\title{
Serodiagnosis of leprosy in patients' contacts by enzyme-linked immunosorbent assay
}

\author{
ELBA GONZALEZ-ABREU*, NANCY MORA $\dagger$, \\ MILAGROS PEREZ†, MARIA PEREIRA $\dagger$, \\ JULIA PEREZ* \& A B GONZALEZ* \\ * Laboratorio de Lepra, Instituto de Medicina Tropical 'Pedro \\ Kouri', Apartado 601, Zona Postal 13, Ciudad de la Habana, Cuba; \\ and $\dagger$ Centro Provincial de Higiene y Epidemiologia, Guantánamo, \\ Cuba
}

\section{Accepted for publication 10 November 1989}

\begin{abstract}
Summary Serum samples from 3336 contacts of leprosy patients were tested for antiphenolic glycolipid I antibodies by enzyme-linked immunosorbent assay with the albumin coupled synthetic disaccharide antigen. The overall positivity rate was $9 \cdot 3 \%$. No significant differences were seen between a group of household contacts of lepromatous patients and those of the other types of the disease. The proportion of ELISA positives was slightly higher in the relatives as compared to workplace contacts and neighbours but significantly different only between the two former $(p<0 \cdot 05)$. Among those contacts with absorbance values higher than $0 \cdot 100,5$ new leprosy patients were diagnosed, 2 of them with positive skin smears. A sixth contact was detected with a very high absorbance value in whom no single skin lesion was found but whose lepromin reaction was $0 \mathrm{~mm}$ and the skin smear showed a bacteriological index of $3+$.
\end{abstract}

\section{Introduction}

It has been suggested that since modern chemotherapy rapidly reduces the number of viable Mycobacterium leprae spread by an individual, the major source of leprosy transmission is likely to be individuals who do not yet have symptoms. ${ }^{1}$ One of the principal goals of leprosy research is to develop tests that will allow identification and early treatment of such individuals, aimed at reducing transmission of the disease. ${ }^{1}$ The incorporation of early diagnosis, especially of multibacillary cases, to the activities of integrated control programmes through appropriate methods of case finding is among the specific approaches considered by WHO to prevent and control leprosy. ${ }^{2}$

However, early diagnosis may not always be easy to attain. Symptoms of leprosy are very often absent or minimal, particularly during the early stages of the disease, hence patients do not voluntarily seek relief through the health care system, ${ }^{3}$ whereas, in other instances, individuals in whom the disease has already manifested do not look for medical care either because of the social stigma or lack of health education. On the other hand, contact surveillance identifies only a small proportion of the total number of new cases 
occurring in a community ${ }^{3}$ and frequently in low endemic areas leprosy is not borne in mind, thus resulting in late diagnosis.

The isolation and chemical characterization of a $M$. leprae species specific antigen, the phenolic glycolipid I (PGI) ${ }^{4,5}$ as well as the preparation of an artificial antigen containing the species specific moiety of $\mathrm{PGI}^{6,7}$ have made the detection and study of anti-M. leprae specific antibodies possible by rather simple serological methods. It has been suggested that these antigens have considerable potential as tools in the serodiagnosis of subclinical stages of leprosy infection, early diagnosis of clinical disease and in detecting transmitters of infection. ${ }^{8-10}$

In this report data are presented from a trial based on the enzyme-linked immunosorbent assay (ELISA) using the albumin coupled synthetic disaccharide (NDA-BSA) antigen to study anti-PGI antibody levels among contacts of leprosy patients and to seek for subclinical transmitters, cases with early clinical stages and individuals at risk of contracting the disease among those presumably infected.

\section{Materials and methods}

SERA

Serum samples from 3336 contacts were tested, of these, 660 were from household contacts, grouped according to the leprosy type (Madrid classification) of the index case, 2134 were relatives in whom no separation into household and non-household or leprosy type of the index case was made, 435 were workplace contacts and 107 were neighbours. They were all living in the province of Guantanamo, Cuba, where leprosy is highly endemic. Venous blood samples were taken, sera obtained and kept frozen at $-20^{\circ} \mathrm{C}$ until used.

ELIS A

The procedure was the same as that described by Cho et al. ${ }^{11}$ except that the antigen was not sonicated but dissolved directly in the buffer and added to wells of flexible PVC plates (Flow Laboratories). The ND-A-BSA antigen was obtained from IMMLEP (Gigg DISSACH). The conjugate reagent was goat anti-human IgM-peroxidase conjugated IgG fraction (Cappel Laboratories). The absorbance was read at $492 \mathrm{~nm}$ in a Titertek Multiskan MC or in an Organon Technika reader. A serum was considered 'positive' when the absorbance exceeded $0 \cdot 160$ since this value corresponded to the 98 th percentile when 100 serum samples obtained from a Havana blood bank were tested to establish the cut-off level.

Dermatological and skin-smear examinations were performed in contacts who showed absorbance values above $0 \cdot 100$ and not in those with lower values. Mitsuda tests with lepromin A, obtained from G. W. Long Hansen's Disease Center, LA 70721, USA, were also included in the clinical study of 200 of such contacts.

Differences between proportions above the cut-off level among the groups of contacts were ascertained by the hypotheses test for two proportions and correlation was also done between the results of ELISA and Mitsuda tests. 


\section{Results}

The overall ELISA positivity rate of all contacts tested was $9 \cdot 3 \%$. Table 1 shows the test results for household contacts grouped according to the leprosy type (Madrid classification) of the index case. No significant differences were seen between the contacts of lepromatous patients and those of the other types of the disease.

The data for other relatives (household and non-household), workplace contacts and neighbours of all leprosy patients are shown in Table 2. The proportion of ELISA positives was slightly higher in the relatives as compared to workplace contacts and neighbours but significantly different only between the two former $(p<0 \cdot 05)$.

The observed distribution of absorbance values is shown in Table 3. Of the total, $81 \cdot 7 \%$ were below $0 \cdot 100,12 \cdot 4 \%$ were in the range $0 \cdot 101-0 \cdot 200,3 \cdot 3 \%$ in the range $0 \cdot 201-$ $0 \cdot 300$ and $2 \cdot 3 \%$ above $0 \cdot 300$. Hypochromic macules found in $2(0 \cdot 5 \%)$ of 417 contacts, in the range $0 \cdot 101-0 \cdot 200$, hypoaesthetic in 1 of them, led to the diagnosis of indeterminate leprosy (patients 1 and 2). Hypochromic macules and a positive Fite-Faraco stained histological section found in $1(4.7 \%)$ of 21 contacts in the range of $0.401-0.500$ led to the diagnosis of the third indeterminate patient. In 1 (50.0\%) of 2 in the range $0 \cdot 701-0 \cdot 800$ clinical signs of lepromatous leprosy and a bacteriological index (BI) of $4+$ were found (patient 4). Two contacts were found with absorbance values above $1 \cdot 000$, one of them with skin lesions and a $\mathrm{BI}$ of $2+$ was diagnosed as a dimorphous leprosy whereas in the other one not a single skin lesion or other dermatological sign was found but the skin smear revealed a BI of $3+$ (patients 5 and 6) Tables 3 and 4.

Mitsuda tests were performed in 200 contacts who showed an absorbance value higher than $0 \cdot 100$. The correlation coefficient $(r)$ was $-0 \cdot 18630$ which is out of the $+/-0 \cdot 13808$ critical interval for a $p=0 \cdot 05$, thus a significant inverse correlation was proved between the lepromin reaction sizes and the absorbance values observed.

Table 1. ELISA results in household contacts grouped according to the leprosy type of the index case

\begin{tabular}{lcr}
\hline $\begin{array}{l}\text { Leprosy type of } \\
\text { the index case* }\end{array}$ & Positive/Total & $\%$ \\
\hline Lepromatous & $38 / 350$ & $10 \cdot 8$ \\
Dimorphous & $12 / 144$ & $8 \cdot 3$ \\
Indeterminate & $10 / 123$ & $8 \cdot 1$ \\
Tuberculoid & $5 / 43$ & $11 \cdot 6$ \\
\hline \multicolumn{3}{c}{$*$ Madrid classification. }
\end{tabular}

Table 2. ELISA results in other contacts of leprosy patients

\begin{tabular}{lcr}
\hline Type of contact & Positive/Total & $\%$ \\
\hline Relatives (household & $216 / 2134$ & $10 \cdot 1$ \\
and non-household) & & \\
Work place contacts & $23 / 435$ & $5 \cdot 2$ \\
Neighbours & $8 / 107$ & $7 \cdot 4$ \\
\hline
\end{tabular}


Table 3. Results of the serological tests, number of skin-smears performed and new cases detected

\begin{tabular}{lccl}
\hline Absorbance level & Sera & Skin smears & \multicolumn{1}{c}{ Cases } \\
\hline$<0 \cdot 100$ & $2728(81 \cdot 7)^{*}$ & NE & NE \\
$0 \cdot 101-0 \cdot 200$ & $417(12 \cdot 4)$ & $213(51 \cdot 0) \dagger$ & $2(0 \cdot 5) \dagger$ \\
$0 \cdot 201-0 \cdot 300$ & $111(3 \cdot 3)$ & $66(59 \cdot 4)$ & 0 \\
$0 \cdot 301-0 \cdot 400$ & $45(1 \cdot 3)$ & $29(64 \cdot 4)$ & 0 \\
$0 \cdot 401-0 \cdot 500$ & $21(0 \cdot 6)$ & $13(61 \cdot 9)$ & $1(4 \cdot 7)$ \\
$0 \cdot 501-0 \cdot 600$ & $7(0 \cdot 2)$ & $5(71 \cdot 4)$ & 0 \\
$0 \cdot 601-0 \cdot 700$ & $3(0 \cdot 08)$ & $3(100 \cdot 0)$ & 0 \\
$0 \cdot 701-0 \cdot 800$ & $2(0 \cdot 08)$ & $2(100 \cdot 0)$ & $1(50 \cdot 0)$ \\
$0 \cdot 801-0 \cdot 900$ & 0 & 0 & 0 \\
$0 \cdot 901-1 \cdot 000$ & 0 & 0 & 0 \\
$>1 \cdot 000$ & $2(0 \cdot 05)$ & $2(100 \cdot 0)$ & $2(100 \cdot 0)$ \\
& & & \\
\hline
\end{tabular}

NE, not examined.

*, percentage of the total number in brackets.

$\dagger$, percentage of the number in the range in brackets.

Table 4. New leprosy cases diagnosed among contacts of leprosy patients

\begin{tabular}{llccl}
\hline & & & \\
Mitsuda & & \\
& Leprosy type* & $(\mathrm{mm})$ & ELISA & BI \\
\hline 1 & Indeterminate & 0 & $0 \cdot 109$ & 0 \\
2 & Indeterminate & 3 & $0 \cdot 125$ & 0 \\
3 & Indeterminate & 5 & $0 \cdot 401$ & 0 \\
4 & Lepromatous & 0 & $0 \cdot 755$ & $4+$ \\
5 & Dimorphous & 0 & $2 \cdot 125$ & $2+$ \\
\hline 6 & Subclinical infection & 0 & $1 \cdot 074$ & $3+$ \\
\hline
\end{tabular}

* Madrid classification.

\section{Discussion}

The ELISA technique with the PGI or its semisynthetic analogues appears to be the most widely used system for the detection of anti- $M$. leprae specific antibodies at present. However not many reports on studies with high risk groups have been published so far. The overall serological positivity rate observed in this study $(9 \cdot 3 \%)$ was lower than that found by other workers in Mexico (23\%) and Sri Lanka (33\%), ${ }^{12}$ but had the same definition of positivity $\left(\mathrm{A}_{492}>0.09\right)$ been used by us it would have been comparable. It was close to those found by Douglas et al. $(11 \cdot 2 \%)$ in Cebu, Philippines ${ }^{13}$ and by Chanteau et al. $(12 \cdot 8 \%)$ in French Polynesia. ${ }^{14}$

Contrary to what was expected, no significant differences were found between the proportions of positive household contacts grouped according to the leprosy type of the index case. It is likely that the contacts of paucibacillary patients may have been exposed 
to the same infection sources of their index cases or to others in the community since they were living in a neighbourhood with a high prevalence rate ( $5 \cdot 6$ per 1000$)$. Seropositivity in the relatives was higher than in the workplace contacts and neighbours but not statistically significant with respect to the latter. The fact that the group of neighbours was represented by persons living on the same block, where as many as 16 patients also lived, might account for this observation since it can be supposed that the risk of infection was high with so many patients in the vicinity.

Previous work with several immunological tests revealed that infection by $M$. leprae is much more frequent than the number of manifest clinical cases would indicate. ${ }^{10}$ While the only accurate way of determining whether the test is useful as a serodiagnostic tool would be to examine clinically and bacteriologically all contacts. In the present study only those with absorbance values above $0 \cdot 100$ were chosen to be studied. The reason was that the work was aimed mainly at assessing the usefulness of the system, by testing serum samples from a rather large number of contacts, for detecting transmitters of infection who might be individuals who did not yet have physical signs and/or cases in very early stages of clinical manifestation, as well as for identifying those presumably infected who might be suspected of incubating multibacillary leprosy and, hence, might be potential transmitters. On the basis of accumulated data, it was assumed that subsequent multiplication of $M$. leprae after infection in such individuals would reach such a bacillary load that it would be reflected in absorbance values higher than $0 \cdot 100$.

In this study the majority of the positive individuals showed low absorbance values which might reflect normal immune responses to $M$. leprae exposures, a few false positive reactions as has been reported ${ }^{6}$ and, in some cases, early stages of infection which could progress towards clinical disease. Much caution should be taken with the small number of contacts exhibiting high absorbance values since they are generally associated with multibacillary leprosy. In this connection the finding of a subclinically infected contact with acid-fast bacilli in his skin smears is of great interest, since this lends support to the assumption that these individuals may play an important role in the transmission of the disease.

It has been reported that this assay is not able to detect a high proportion of paucibacillary patients without considerable loss of specificity. ${ }^{15}$ However, the usefulness of the system as a test for leprosy infection and its use for control purposes should be discussed in relation to particular epidemiological situations and to the quality of the control programme. There is evidence that the distribution of the different types of the disease may vary between different populations. ${ }^{16}$ In Cuba, the proportion of multibacillary leprosy (lepromatous and dimorphous in the Madrid classification) reaches about $50 \%$ of the annually detected cases. ${ }^{17}$ Therefore, in areas with a relatively high prevalence of multibacillary leprosy, the value of this tool for screening purposes may be important in terms of leading to the detection of a number of early cases and the identification of presumably infected individuals who could be examined and followed up. In addition, the administration of chemoprophylaxis to these latter subjects could also be evaluated.

\section{Acknowledgments}

This investigation received financial support from the UNDP/World Bank/WHO Special Programme for Research and Training in Tropical Diseases. 


\section{References}

1 Anonymous. Serological test for leprosy. Lancet, 1986; i: 533-5.

2 Anonymous. Eighth General Programme of Work. Geneva: World Health Organization, 1987; 'Health for all' Series No. 10.

3 WHO. A Guide to Leprosy Control. Second edition. World Health Organization, Geneva, 1988.

${ }^{4}$ Hunter SW, Brennan PJ. A novel phenolic glycolipid from Mycobacterium leprae possible involved in immunogenicity and pathogenicity. J Bacteriol, 1981; 147: 728-35.

5 Hunter SW, Fujiwara T, Brennan PJ. Structure and antigenicity of the major specific glycolipid antigen of Mycobacterium leprae. J Biol Chem, 1982; 257: 15072-8.

6 Cho SN, Fujiwara T, Hunter SW, Rea TH, Gelber RH, Brennan PJ. Use of an artificial antigen containing the 3,6-di-O-methyl-B-D-pyranosyl epitope for the serodiagnosis of leprosy. J Infect Dis, 1984; 150: 311-22.

7 Fujiwara T, Hunter SW, Cho SN, Aspinall GO, Brennan PJ. Chemical synthesis and serology of the disaccharides and trisaccharides of phenolic glycolipid antigens from the leprosy bacillus and preparation of disaccharide protein conjugate for serodiagnosis of leprosy. Infect Immun. 1984; 43: 245-52.

8 Young DB, DissanayakeS, Miller RA, Khanolkar SR, Buchanan TM. Humans respond predominantly with IgM immunoglobulin to the species-specific glycolipid of Mycobacterium leprae. J Infect Dis, 1984; 149; 8703.

9 Ralhan R, Band AH, Roy A, Hajini GH, Sharma AK, Talwar GP. An enzyme immunoassay titrating IgM antibody against phenolic glycolipid for diagnosis of lepromatous leprosy. Ind J Med Res, 1985; 82: 110-15.

10 Grupo de Estudio de la OMS. Epidemiologia de la lepra en relación con la lucha antileprosa. Ginebra: Organización Mundial de la Salud, 1985; Serie de Informes Técnicos 716.

11 Cho SN, Yanagihara DL, Hunter SW, Gelber RH, Brennan PJ. Serological specificity of phenolic glycolipid I from Mycobacterium leprae and use in serodiagnosis of leprosy. Infect Immun, 1983; 41: 1077-83.

12 Buchanan TM, Dissanayake S, Young DB. Evaluation of the significance of antibodies to phenolic glycolipid of Mycobacterium leprae in leprosy patients and their contacts. Int J Lepr, 1983; 51: 658-9.

13 Douglas JT, Celona RV, Abalos RM, Madarang MG, Fajardo T. Serological reactivity and early detection of leprosy among contacts of lepromatous patients in Cebu, The Philippines. Int J Lepr, 1987; 55: 718-21.

14 Chateau S, Cartel J-L, Guidi C, Plichart R, Bach M-A. Seroepidemiological study on 724 household contacts of leprosy patients in French Polynesia using disaccharide-octyl-BSA as antigen. Int J Le pr, 1987; 55: 626-32.

15 Burgess PJ, Fine PEM, Ponnighaus JM, Draper C. Serological tests in leprosy. The sensitivity, specificity and predictive value of ELISA tests based on phenolic glycolipid antigens, and the implications for their use in epidemiological studies. Epidemiol Inf, 1988; 101: 159-71.

16 Fine PEM. Leprosy: The epidemiology of a slow bacterium. Epidemiologic Rev, 1982; 4: 161-88.

17 Gil RE. Situacioń de la lepra en Cuba. Dirección Nacional de Epidemiología. Ministerio de Salud Pública. Ciudad Habana. 1988. 